\title{
On the quadratic moment of self-normalized sums
}

Fredrik Jonsson

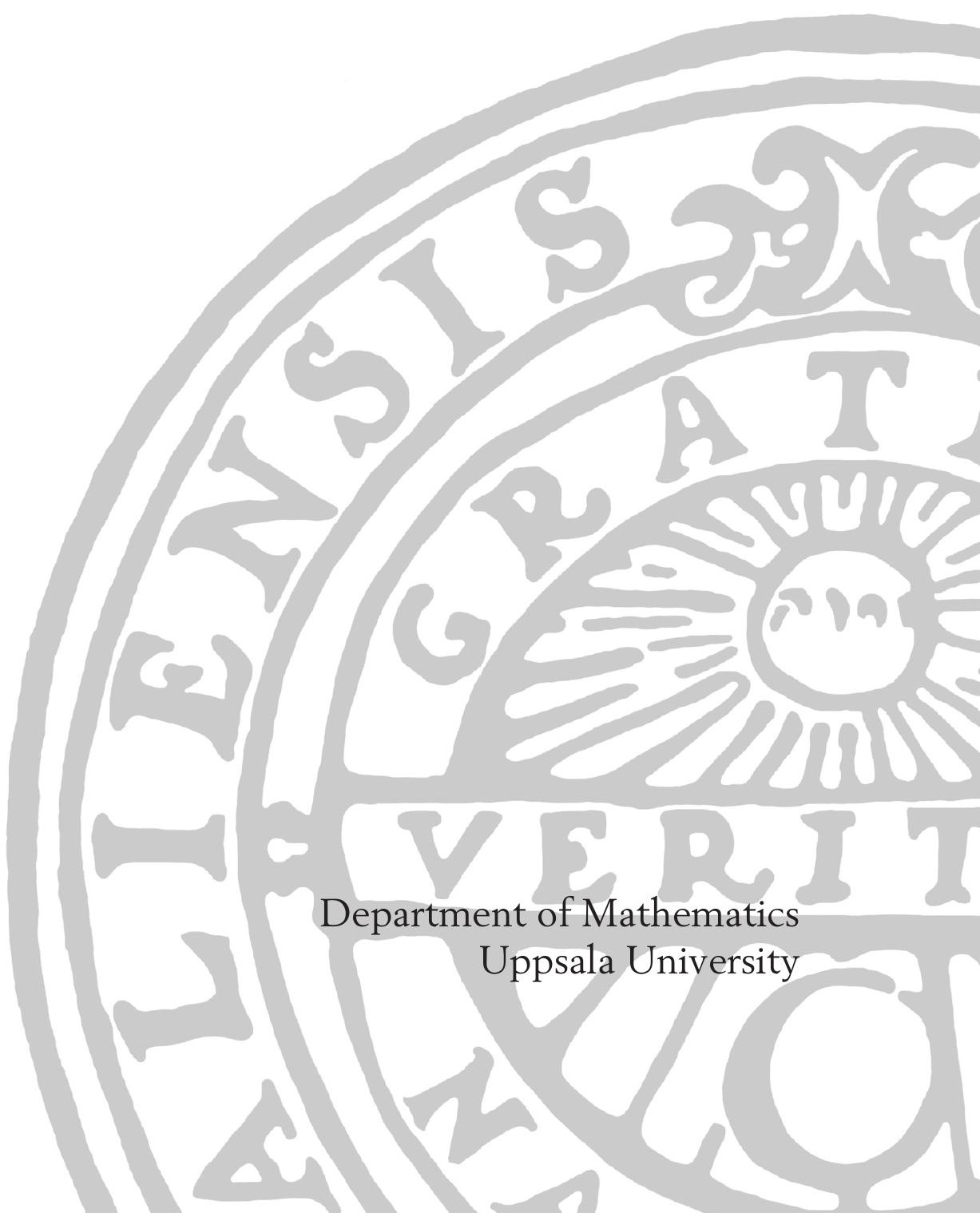





\title{
On the quadratic moment of self-normalized sums
}

\author{
Fredrik Jonsson \\ Uppsala University
}

\begin{abstract}
Let an integer $n \geq 2$ and a vector of independent, identically distributed random variables $\mathbf{X}=\left(X_{1}, \ldots, X_{n}\right)$ be given with $\mathrm{P}(\mathbf{X}=\mathbf{0})=0$ and define the self-normalized sum $Z_{n}=$ $\left(\sum_{i=1}^{n} X_{i}\right) /\left(\sum_{i=1}^{n} X_{i}^{2}\right)^{1 / 2}$. We derive a formula for $\mathrm{E} Z_{n}^{2}$ which enables us to prove that $\mathrm{E} Z_{n}^{2} \geq$ 1 and that $\mathrm{E} Z_{n}^{2}=1$ if and only if the summands are symmetrically distributed. The formula moreover suggests nonparametric estimators of $\mathrm{E} Z_{n}^{2}$ given $\mathbf{X}$ which we comment upon. We also construct examples where $Z_{n}$ converges to the standard normal distribution as $n$ tends to infinity while $\mathrm{E} Z_{n}^{2}$ tends to infinity (the distribution of the summands varies with $n$ ).
\end{abstract}

\section{Introduction}

A wellknown problem in statistical theory and practice is the following: Observations $x_{1}, \ldots, x_{n}$ (an i.i.d. sample) are given (random variation centered at value $\mu$ ). Of what kind and precision is our information about $\mu$ ? There is also a wellknown solution approach: assume that the distribution of the sample is $\mathrm{N}\left(\mu, \sigma^{2}\right)$ with a positive parameter $\sigma$ modelling the unknown magnitude of random variation. Then consider the concepts of significance testing and confidence interval with respect to $\mu$. A reasonable form of the latter is in this case $\bar{x} \pm t \hat{\sigma}_{n} / \sqrt{n}$, with $\pm t$ being the values of symmetric truncation with respect to a chosen level of confidence of the distribution of the studentized statistic $T_{n}$,

$$
T_{n}=\frac{S_{n}-n \mu}{\sqrt{n} \hat{\sigma}_{n}} \quad \text { with } \quad \hat{\sigma}_{n}^{2}=\frac{1}{n-1} \sum_{i=1}^{n}\left(X_{i}-S_{n} / n\right)^{2} \quad \text { and } \quad S_{n}=\sum_{i=1}^{n} X_{i}
$$

Practioners and theoretical statisticians have been motivated during the last hundred years to consider questions related to the replacement of $\mathrm{N}\left(\mu, \sigma^{2}\right)$ by some other scale-location family of distributions, meaning that the distribution function of the sample is given by $F_{\mu, \sigma}(x)=F((x-$ $\mu) / \sigma$ ) with respect to some "standardized" distribution function $F$. Typical questions are: How are $p$-values in significance testing and confidence levels in the confidence interval approach affected by non-normality? What shall I assume of $F$ and the sample size for the effect to be negligible? Based upon the sample, can I judge whether $F$ is sufficiently normal-like? Are there alternative methods (strong and wellfounded candidates) if I feel uncomfortable with the normal assumption approach?

The present work is motivated by the belief that important contributions to these kind of concerns still can be made. We focus in particular on the first two questions. Further motivation was provided by the suggestion that disturbance of $p$-values in practice usually is connected with pronounced skewness in the underlying distribution (if the i.i.d. property is not violated). Confer [4] and [10] for a review on research related to this suggestion. Posten [8] renders certain support to the hypothesis by reporting on simulation results with respect to distributions from the so called Pearson system of distributions with varying skewness and kurtosis.

A second incitament to the present work is the article [5] by Efron which provides results relating to the claim that $T_{n}$ is well concentrated around the origin in cases where skewness is completely absent, i.e. when the sampling distribution is symmetric around the origin $(X \stackrel{d}{=}-X)$.

AMS 2000 subject classifications. Primary 60E05, 62F35; Secondary 62G05.

Keywords and phrases. quadratic moment, self-normalization, symmetric distributions, robustness, student's $t$-test. Abbreviated title. On the quadratic moment of self-normalized sums.

Date. October 26, 2009 
An important role is played by the observation that $T_{n}$ (assume from now on that $\mu=0$ in the definition of $T_{n}$ above) is intimately related to the self-normalized sum $Z_{n}$,

$$
Z_{n}=S_{n} / V_{n} \quad \text { with } \quad V_{n}=\left(\sum_{i=1}^{n} X_{i}^{2}\right)^{1 / 2} \quad \text { and } \quad S_{n}=\sum_{i=1}^{n} X_{i} .
$$

Indeed, the monotone relationship can be spelled out as

$$
T_{n}=Z_{n} \sqrt{(n-1) /\left(n-Z_{n}^{2}\right)}, \quad Z_{n}=T_{n} \sqrt{n /\left(n-1+T_{n}^{2}\right)} .
$$

A consequence is that the concentrations of $Z_{n}$ and $T_{n}$ around the origin are closely related, especially for moderate and large values of $n$. Surprisingly low upper bounds for even moments $\mathrm{E} Z_{n}^{2 k}$ were derived in [5] under the assumption of symmetry. The case $k=1$ is of a special kind since $\mathrm{E} Z_{n}^{2}$ is equal to one under the symmetry assumption (cf. [5]). We were thereby tempted to ask: Is there any distribution function $F$ and any $n \geq 2$ such that $\mathrm{E} Z_{n}^{2}$ is smaller than one? The answer turns out to be negative. We moreover show that $\mathrm{E} Z_{n}^{2}=1$ characterizes the symmetry assumption (so that symmetry in a certain sense is optimal for having $T_{n}$ concentrated around the origin). In other words:

$$
\mathrm{E} Z_{n}^{2} \geq 1 \quad \text { with } \quad \mathrm{E} Z_{n}^{2}=1 \quad \text { iff } \quad X \stackrel{d}{=}-X .
$$

Note however that $T_{n}$ may be far from $t$-distributed even for distributions symmetric around the origin. An introduction and discussion of symmetric (as well as asymmetric) distributions with an arbitrarily small variation of the corresponding $T_{n}^{2}$ around 1 is given in the appendix.

\section{A summary of the paper}

Section 3 presents a formula for $\mathrm{E} Z_{n}^{2}$ with $\mathrm{E} Z_{n}^{2} \geq 1$ as an immediate consequence. Section 4 shows that $\mathrm{E} Z_{n}^{2}=1$ and symmetric sampling distribution coincide under the i.i.d. assumption.

The results in Section 5 were motivated by the concern: For which non-symmetric distributions is $\mathrm{E} Z_{n}^{2}-1$ small? An investigation of the character of $\lim _{m \rightarrow \infty} \mathrm{E} Z_{n, m}^{2}=1$ with respect to families of distribution functions $\left\{F_{m}\right\}_{m \geq 1}$ is presented. The answer to the question turns out to be somewhat more complicated than "the nearly symmetric distributions".

At issue in Section 6 is a comparison of the behaviour of $\mathrm{E} Z_{n}^{2}$ as $n$ tends to infinity with the accuracy of the normal approximation of $Z_{n}$. An analysis with respect to mean-zero two-point distributions with increasing degree of asymmetry is presented. One conclusion is that cases of growing asymmetry exist where $\mathrm{E} Z_{n}^{2}$ diverges to infinity while nevertheless normal convergence of $Z_{n}$ takes place.

Section 7 suggests, based upon the formula in Section 3 , an estimator of $\mathrm{E} Z_{n}^{2}$ given $\mathbf{X}=$ $\left(X_{1}, \ldots, X_{n}\right)$ and an estimator of $\mathrm{E} Z_{n}^{2}$ with respect to a mean-zero probability distribution and observations from the same distribution shifted by some unknown constant. Some basic properties of the two estimators are derived and discussed.

Distributions with corresponding $T_{n}^{2}$ almost constantly equal to 1 are finally introduced and discussed in the appendix. This phenomenon has an impact on the results of Section 5 and seems to be interesting in its own right.

\section{A formula for $\mathrm{E} Z_{n}^{2}$}

The present and the next section concern vectors $\mathbf{X}=\left(X_{1}, \ldots, X_{n}\right), X_{i} \stackrel{d}{=} X, n \geq 2$, of independent and identically distributed random variables, where $X$ has distribution function $F$ continuous at the origin. The self-normalized sum $Z_{n}$ is then defined as in the introduction (welldefined almost surely by the continuity assumption on $F$ ).

Theorem 3.1. For all $n$ and $F$,

$$
\mathrm{E} Z_{n}^{2}=1+\left(n^{2}-n\right) \int_{0}^{\infty}\left(\mathrm{E}\left(X e^{-t X^{2}}\right)\right)^{2}\left(\mathrm{E} e^{-t X^{2}}\right)^{n-2} \mathrm{~d} t
$$


Corollary 3.1. $\mathrm{E} Z_{n}^{2} \geq 1$ for all $n$ and $F$.

Lemma 3.1. For all $n$ and $F$,

$$
\mathrm{E}\left(X_{1} X_{2} / \sum_{k=1}^{n} X_{k}^{2}\right)=\int_{0}^{\infty}\left(\mathrm{E}\left(X e^{-t X^{2}}\right)\right)^{2}\left(\mathrm{E} e^{-t X^{2}}\right)^{n-2} \mathrm{~d} t .
$$

Proof of Lemma 3.1. For any $a>0, a^{-1}=\int_{0}^{\infty} e^{-t a} \mathrm{~d} t$, so that

$$
\begin{aligned}
\mathrm{E}\left(X_{1} X_{2} / \sum_{k=1}^{n} X_{k}^{2}\right) & =\mathrm{E}\left(X_{1} X_{2} \int_{0}^{\infty} e^{-t \sum X_{k}^{2}} \mathrm{~d} t\right) \\
& =\mathrm{E}\left(\int_{0}^{\infty} X_{1} e^{-t X_{1}^{2}} X_{2} e^{-t X_{2}^{2}} e^{-t \sum_{k=3}^{n} X_{k}^{2}} \mathrm{~d} t\right) .
\end{aligned}
$$

It then only remains, due to independence, to verify that

$$
\begin{aligned}
& \mathrm{E}\left(\int_{0}^{\infty} X_{1} e^{-t X_{1}^{2}} X_{2} e^{-t X_{2}^{2}} e^{-t \sum_{k=3}^{n} X_{k}^{2}} \mathrm{~d} t\right) \\
= & \int_{0}^{\infty} \mathrm{E}\left(X_{1} e^{-t X_{1}^{2}} X_{2} e^{-t X_{2}^{2}} e^{-t \sum_{k=3}^{n} X_{k}^{2}}\right) \mathrm{d} t .
\end{aligned}
$$

Set $\mathbf{x}=\left(x_{1}, \ldots, x_{n}\right)$ and $g(\mathbf{x}, t)=x_{1} x_{2} \exp \left(-t \sum_{k=1}^{n} x_{k}^{2}\right)$. Identity (1) follows from the Fubini theorem, ([3, Proposition 5.2.1., page 159]), since

$$
\mathrm{E}\left(\int_{0}^{\infty}\left|X_{1}\right| e^{-t X_{1}^{2}}\left|X_{2}\right| e^{-t X_{2}^{2}} e^{-t \sum_{k=3}^{n} X_{k}^{2}} \mathrm{~d} t\right)=\mathrm{E}\left(\left|X_{1} X_{2}\right| / \sum_{k=1}^{n} X_{k}^{2}\right) \leq 1,
$$

and since $g$ is continuous and thereby measurable.

Proof of Theorem 3.1. Expand $Z_{n}^{2}$ :

$$
Z_{n}^{2}=\left(\sum_{i=1}^{n} X_{i}\right)^{2} / \sum_{k=1}^{n} X_{k}^{2}=1+\sum_{i \neq j} X_{i} X_{j} / \sum_{k=1}^{n} X_{k}^{2}
$$

It follows that

$$
\mathrm{E} Z_{n}^{2}=1+\left(n^{2}-n\right) \mathrm{E}\left(X_{1} X_{2} / \sum_{k=1}^{n} X_{k}^{2}\right)
$$

since $X_{1}, \ldots, X_{n}$ are identically distributed. Then apply Lemma 3.1.

\section{Characterizing $\mathrm{E} Z_{n}^{2}=1$}

It was noted by Efron in [5] that $\mathrm{E} Z_{n}^{2}=1$ in case all summands are symmetrically distributed (not necessarily identically distributed). With Theorem 3.1 as our point of departure we now extend this result (for the restricted case of i.i.d. summands) to the following theorem.

Theorem 4.1. The following two conditions are equivalent (with respect to any $n \geq 2$ ):

$$
\text { (i) } \quad \mathrm{E} Z_{n}^{2}=1 ; \quad \text { (ii) } X \text { is symmetrically distributed }(X \stackrel{d}{=}-X) \text {. }
$$

Proof. It is clear that $\mathrm{E} Z_{n}^{2}=1$ if $F$ is symmetric, e.g. in view of Theorem 3.1 , since $\mathrm{E}\left(X e^{-t X^{2}}\right)=0$ in case of symmetry. It hence remains to prove that $\mathrm{E} Z_{n}^{2}=1$ implies symmetry, i.e. that

$$
\mathrm{E}\left(X_{1} X_{2} / \sum_{k=1}^{n} X_{k}^{2}\right)=0 \Longrightarrow X \stackrel{d}{=}-X .
$$

Apply Lemma 3.1 to rewrite the left hand side to

$$
\int_{0}^{\infty}\left(\mathrm{E}\left(X e^{-t X^{2}}\right)\right)^{2}\left(\mathrm{E} e^{-t X^{2}}\right)^{n-2} \mathrm{~d} t=0,
$$


which is equivalent to

$$
\mathrm{E}\left(X e^{-t X^{2}}\right)=0, \quad \text { all } t>0,
$$

since the integrand is nonnegative and continuous in $t$. Define random variables

$$
X^{+}=X \cdot I\{X>0\}, \quad X^{-}=-X \cdot I\{X<0\} .
$$

We may then write

$$
\mathrm{E}\left(X e^{-t X^{2}}\right)=\mathrm{E}\left(X^{+} e^{-t\left(X^{+}\right)^{2}}\right)-\mathrm{E}\left(X^{-} e^{-t\left(X^{-}\right)^{2}}\right),
$$

and it suffices to prove that

$$
\mathrm{E}\left(X^{+} e^{-t\left(X^{+}\right)^{2}}\right)=\mathrm{E}\left(X^{-} e^{-t\left(X^{-}\right)^{2}}\right) \quad \text { for all } \quad t>0 \Longrightarrow X^{+} \stackrel{d}{=} X^{-} .
$$

The left hand side in (2) implies (by linearity) that

$$
\mathrm{E} g\left(X^{+}\right)=\mathrm{E} g\left(X^{-}\right) \quad \text { for all functions } g(x)=\sum_{k=1}^{n} a_{k} x e^{-k x^{2}} \text { with } n \geq 1 \text { and } a_{k} \in \mathbb{R},
$$

while the right hand side follows from elementary analytical arguments and

$$
\operatorname{E} f\left(X^{+}\right)=\operatorname{E} f\left(X^{-}\right) \quad \text { for all } f \in C([0, \infty)) \text { with supp } f \subset[a, b] \text { for some } 0<a<b<\infty .
$$

We conclude that (2) is established by showing that (3) implies (4). For this it suffices to establish the possibility of uniform approximation of a function $f \in \mathcal{F}$ by functions $g \in \mathcal{G}$, which is shown to be a consequence of a famous theorem of Weierstrass in the following lemma.

Lemma 4.1. Every function $f \in \mathcal{F}$ can be uniformly approximated with functions $g \in \mathcal{G}$ :

$$
\begin{aligned}
& \mathcal{F}=\{f \in C([0, \infty)): \operatorname{supp} f \subset[a, b], \text { for some } 0<a<b<\infty\}, \\
& \mathcal{G}=\left\{g \in C([0, \infty)): g(x)=\sum_{k=1}^{n} a_{k} x e^{-k x^{2}} \text { for some } n \geq 1 \text { and some } a_{k} \in \mathbb{R}\right\} .
\end{aligned}
$$

Proof. Let $f \in \mathcal{F}$ be given and assume candidates $g_{j} \in \mathcal{G}$. Setting $p_{j}(y)=g_{j}(x) / x$, with the change of variables $y=e^{-x^{2}}$, yields

$$
\sup _{x \geq 0}\left|g_{j}(x)-f(x)\right|=\sup _{0<y \leq 1}\left|\sqrt{\log (1 / y)} \cdot p_{j}(y)-f(\sqrt{\log (1 / y)})\right| .
$$

Note that $p_{j}$ is an arbitrary polynomial of degree $\geq 1$ with no constant term. Rewrite (5) as

$$
\sup _{0<y \leq 1}\left|y \sqrt{\log (1 / y)} \cdot p_{j}^{*}(y)-f(\sqrt{\log (1 / y)})\right|
$$

with $p_{j}^{*}(y)=p_{j}(y) / y$. Define $f^{*} \in C[0,1]$ through the relation

$$
f(\sqrt{\log (1 / y)})=y \sqrt{\log (1 / y)} \cdot f^{*}(y),
$$

which is possible since $y \mapsto f(\sqrt{\log (1 / y)})$ has support within an interval $[\varepsilon, 1-\varepsilon]$, for some $\varepsilon>0$, and since $y \mapsto y^{-1}(\log (1 / y))^{-1 / 2}$ is welldefined and continuous within such an interval. Now

$$
\sup _{0<y \leq 1}\left|y \sqrt{\log (1 / y)} \cdot p_{j}^{*}(y)-y \sqrt{\log (1 / y)} \cdot f^{*}(y)\right|=\sup _{0 \leq y \leq 1} y \sqrt{\log (1 / y)}\left|p_{j}^{*}(y)-f^{*}(y)\right|,
$$

since $\lim _{y \rightarrow 0} y \sqrt{\log (1 / y)}=0$. The theorem of Weierstrass ([9, Theorem 7.26, page 159]) yields the uniform approximation of $f^{*}$ by functions $p_{j}^{*}$, since $f^{*} \in C[0,1], p_{j}^{*}$ is an arbitrary polynomial, and since $y \mapsto y \sqrt{\log (1 / y)}$ is bounded on $[0,1]$, which finishes the proof. 


\section{On the characterization of $\lim _{m \rightarrow \infty} \mathrm{E} Z_{n, m}^{2}=1$}

Consider a sequence of cumulative distribution functions $\left\{F_{m}\right\}_{m \geq 1}$ continuous at the origin and define

$$
Z_{n, m}=\left(\sum_{i=1}^{n} X_{i, m}\right) /\left(\sum_{i=1}^{n} X_{i, m}^{2}\right)^{1 / 2}
$$

with respect to $n$ independent random variables $X_{1, m}, \ldots, X_{n, m}$ each with probability distribution defined by $F_{m}$. Moreover, with respect to a random variable $X$ with probability distribution $\nu$ and cumulative distribution function $F$, define $\nu^{+}$and $\nu^{-}$as the probability distributions of $X^{+}=X \cdot I\{X>0\}$ and $X^{-}=-X \cdot I\{X<0\}$, respectively, and $F^{+}$and $F^{-}$as the corresponding distribution functions.

Theorem 4.1 stated that equality $\mathrm{E} Z_{n}^{2}=1$ is equivalent to the distributional equality $\nu^{+}=\nu^{-}$ (independently of $n \geq 2$ ). One could therefore conjecture that convergence $\lim _{m \rightarrow \infty} \mathrm{E} Z_{n, m}^{2}=1$ is equivalent to the distributional convergence

$$
\lim _{m \rightarrow \infty} \nu_{m}^{+}-\nu_{m}^{-} \stackrel{d}{=} 0
$$

the latter understood in a weak convergence sense, i.e.

$$
\lim _{m \rightarrow \infty} \int_{0}^{\infty} g(x) \mathrm{d} F_{m}^{+}(x)-\int_{0}^{\infty} g(x) \mathrm{d} F_{m}^{-}(x)=0 \quad \text { for all } g \in C_{b}\left(\mathbb{R}_{>0}\right) .
$$

This conjecture is however not true, Example 8.1 and Theorem 8.1 in the appendix reveal that the property of distributional convergence is not necessary for convergence of moments. The example seems to be characteristic regarding a sense in which probability mass escapes to zero (and/or infinity). The conjecture turns out to be true if such behaviour is excluded by imposing tightness of $\left\{\nu_{m}\right\}$ with respect to $\mathbb{R} \backslash\{0\}$.

Definition 5.1. (cf. [2, Section 5, page 58]) A sequence $\left\{\nu_{m}\right\}$ of probability distributions on $\mathbb{R}$ is said to be tight with respect to $\mathbb{R} \backslash\{0\}$ if for each $\varepsilon>0$ there exists $0<\delta<1$, such that

$$
\nu_{m}(\{x: \delta<|x|<1 / \delta\})>1-\varepsilon \quad \text { for all } m \geq 1 .
$$

Theorem 5.1. For sequences $\left\{\nu_{m}\right\}$ of probability distributions that are tight with respect to $\mathbb{R} \backslash\{0\}$ and integers $n \geq 2$, the following two conditions are equivalent:

$$
\text { (i) } \quad \lim _{m \rightarrow \infty} \mathrm{E} Z_{n, m}^{2}=1 ; \quad \text { (ii) } \quad \lim _{m \rightarrow \infty} \nu_{m}^{+}-\nu_{m}^{-} \stackrel{d}{=} 0 .
$$

Proof. First assume that $\nu_{m}^{+}-\nu_{m}^{-} \stackrel{d}{\longrightarrow} 0$, i.e. that there exists a function $g \in C_{b}\left(\mathbb{R}_{>0}\right)$, a subsequence $\left\{m_{k}\right\}$ and a number $\delta>0$ such that

$$
\left|\int_{0}^{\infty} g(x) \mathrm{d} F_{m_{k}}^{+}(x)-\int_{0}^{\infty} g(x) \mathrm{d} F_{m_{k}}^{-}(x)\right|>\delta \quad \text { for all } \quad k \geq 1 .
$$

The assumption of tightness and Prohorov's theorem, [2, Theorem 5.1, page 59], gives a further subsequence $\left\{m_{k_{l}}\right\}$ and a probability distribution $\mu$ with respect to $\mathbb{R} \backslash\{0\}$ such that $\mu_{m_{k_{l}}} \stackrel{d}{\longrightarrow} \mu$. It follows that, for any $t>0$,

$$
\begin{aligned}
& \lim _{l \rightarrow \infty}\left(\int_{-\infty}^{\infty} x e^{-t x^{2}} \mathrm{~d} F_{m_{k_{l}}}(x)\right)^{2}\left(\int_{-\infty}^{\infty} e^{-t x^{2}} \mathrm{~d} F_{m_{k_{l}}}(x)\right)^{n-2} \\
= & \left(\int_{-\infty}^{\infty} x e^{-t x^{2}} \mathrm{~d} F(x)\right)^{2}\left(\int_{-\infty}^{\infty} e^{-t x^{2}} \mathrm{~d} F(x)\right)^{n-2}
\end{aligned}
$$

and from (7) that $\mu^{+} \neq \mu^{-}$. We conclude upon these two facts, Theorem 3.1, Theorem 4.1 and Fatou's lemma that

$$
\begin{aligned}
& \liminf _{l \rightarrow \infty} \mathrm{E} Z_{n, m_{k_{l}}}^{2} \\
= & 1+\left(n^{2}-n\right) \liminf _{l \rightarrow \infty} \int_{0}^{\infty}\left(\int_{-\infty}^{\infty} x e^{-t x^{2}} \mathrm{~d} F_{m_{k_{l}}}(x)\right)^{2}\left(\int_{-\infty}^{\infty} e^{-t x^{2}} \mathrm{~d} F_{m_{k_{l}}}(x)\right)^{n-2} \mathrm{~d} t . \\
\geq & 1+\left(n^{2}-n\right) \int_{0}^{\infty}\left(\int_{-\infty}^{\infty} x e^{-t x^{2}} \mathrm{~d} F(x)\right)^{2}\left(\int_{-\infty}^{\infty} e^{-t x^{2}} \mathrm{~d} F(x)\right)^{n-2} \mathrm{~d} t>1,
\end{aligned}
$$


and consequently that $\mathrm{E} Z_{n, m}^{2} \nrightarrow 1$ as $m \rightarrow \infty$.

Next assume that $\nu_{m}^{+}-\nu_{m}^{-} \stackrel{d}{\longrightarrow} 0$. It suffices to prove that, for each subsequence $\left\{m_{k}\right\}$, there exists a further subsequence $\left\{m_{k_{l}}\right\}$ such that $\lim _{l \rightarrow \infty} \mathrm{E} Z_{n, m_{k_{l}}}^{2}=1$ (the principle of subsequences). Given $\left\{m_{k}\right\}$, apply the assumption of tightness and Prohorov's theorem to arrive at a subsequence $\left\{m_{k_{l}}\right\}$ and a probability distribution $\nu$ such that $\nu_{m_{k_{l}}} \stackrel{d}{\longrightarrow} \nu$. Now $\nu^{+}=\nu^{-}$, since the converse contradicts the initial assumption. It suffices (cf. Theorem 3.1) to show that

$$
\lim _{k \rightarrow \infty} \int_{0}^{\infty}\left(\int_{-\infty}^{\infty} x e^{-t x^{2}} \mathrm{~d} F_{m_{k_{l}}}(x)\right)^{2}\left(\int_{-\infty}^{\infty} e^{-t x^{2}} \mathrm{~d} F_{m_{k_{l}}}(x)\right)^{n-2} \mathrm{~d} t=0 .
$$

Assume without loss of generality that $n=2$, i.e. replace the target equation (9) with

$$
\lim _{k \rightarrow \infty} \int_{0}^{\infty}\left(\int_{-\infty}^{\infty} x e^{-t x^{2}} \mathrm{~d} F_{m_{k_{l}}}(x)\right)^{2} \mathrm{~d} t=0 .
$$

Set $A_{M}=(0,1 / M) \cup(M, \infty)$. The assumption of tightness yields that, for any $\varepsilon>0$, there exists $M$ such that

$$
\int_{A_{M}} \mathrm{~d} F_{m_{k_{l}}}^{+}(x)<\varepsilon, \quad \int_{A_{M}} \mathrm{~d} F_{m_{k_{l}}}^{-}(x)<\varepsilon \quad \text { for all } \quad l \geq 1 .
$$

Then, due to Cauchy-Schwarz inequality, Fubini's theorem and (11),

$$
\begin{aligned}
& \int_{0}^{\infty}\left(\int_{|x| \in A_{m}} x e^{-t x^{2}} \mathrm{~d} F_{m_{k_{l}}}\right)^{2} \mathrm{~d} t \\
= & \int_{0}^{\infty}\left(\int_{A_{M}} x e^{-t x^{2}} \mathrm{~d} F_{m_{k_{l}}}^{+}(x)-\int_{A_{M}} x e^{-t x^{2}} \mathrm{~d} F_{m_{k_{l}}}^{-}(x)\right)^{2} \mathrm{~d} t \\
\leq & \int_{0}^{\infty}\left(\int_{A_{M}} x e^{-t x^{2}} \mathrm{~d} F_{m_{k_{l}}}^{+}(x)\right)^{2}+\left(\int_{A_{M}} x e^{-t x^{2}} \mathrm{~d} F_{m_{k_{l}}}^{-}(x)\right)^{2} \mathrm{~d} t \\
\leq & \int_{0}^{\infty}\left(\int_{A_{M}} x^{2} e^{-2 t x^{2}} \mathrm{~d} F_{m_{k_{l}}}^{+}(x) \int_{A_{M}} \mathrm{~d} F_{m_{k_{l}}}^{+}(x)+\int_{A_{M}} x^{2} e^{-2 t x^{2}} \mathrm{~d} F_{m_{k_{l}}}^{-}(x) \int_{A_{M}} \mathrm{~d} F_{m_{k_{l}}}^{-}(x)\right) \mathrm{d} t \\
= & \left(\int_{A_{M}} \mathrm{~d} F_{m_{k_{l}}}^{+}(x)\right)^{2} / 2+\left(\int_{A_{M}} \mathrm{~d} F_{m_{k_{l}}}^{-}(x)\right)^{2} / 2<\varepsilon^{2} .
\end{aligned}
$$

In proving

$$
\lim _{l \rightarrow \infty} \int_{0}^{\infty}\left(\int_{|x| \notin A_{m}} x e^{-t x^{2}} \mathrm{~d} F_{m_{k_{l}}}\right)^{2} \mathrm{~d} t=0 \quad \text { for almost all } \quad M>0,
$$

we first note that

$$
\lim _{l \rightarrow \infty} \int_{|x| \notin A_{m}} x e^{-t x^{2}} \mathrm{~d} F_{m_{k_{l}}}=\int_{|x| \notin A_{m}} x e^{-t x^{2}} \mathrm{~d} F=0 \quad \text { for almost all } \quad M>0,
$$

since $F$ has countably many discontinuity points, $F^{+}=F^{-}$, and in view of the mapping theorem, [2, Theorem 2.7, page 21]. We next apply (14) in Lebesgue's dominated convergence theorem, [3, Theorem 2.4.4, page 72], with respect to integration $(\mathrm{d} t)$ over the positive real line. This is justified by the simple relations

$$
\left(\int_{|x| \notin A_{m}} x e^{-t x^{2}} \mathrm{~d} F_{m_{k_{l}}}\right)^{2} \leq M^{2} e^{-t / M^{2}}, \quad \int_{0}^{\infty} M^{2} e^{-t / M^{2}} \mathrm{~d} t=M^{4},
$$

and yields (13). We conclude that (10) holds in view of (12) and (13), since $\varepsilon$ was arbitrary.

\section{On the behaviour of $\mathrm{E} Z_{n}^{2}$ as $n$ tends to infinity}

A sequence of self-normalized sums $\left\{Z_{n}\right\}_{n \geq 1}$ converges to the standard normal distribution as $n \rightarrow \infty$ with $F$ fixed if and only if $\mathrm{E} X=0$ and $\left\{S_{n} / a_{n}\right\}$ converges to the normal distribution, 
for some choice of normalizing sequence $\left\{a_{n}\right\}$. This was proved by Giné, Götze and Mason in [6], where it was also shown that if $\left\{Z_{n}\right\}$ is tight then it is subgaussian, in the sense that

$$
\mathrm{E} \exp \left(t Z_{n}\right) \leq 2 \exp \left(c t^{2}\right), \quad \text { for some constant } c \text { and all } n \text {. }
$$

A consequence of the subgaussian property is that $\left\{\mathrm{E} Z_{n}^{2}\right\}$ converges to 1 whenever $\left\{Z_{n}\right\}$ converges to the normal distribution (cf. [7, Theorems 4.2 and 5.9, Chapter 5]). Note that the latter very well may occur for highly non-symmetric distributions (zero mean and finite second moment is sufficient), whereas convergence of $\left\{\mathrm{E} Z_{n, m}^{2}\right\}_{m}$ to 1 typically occurs for distributions with increasing degree of symmetry (cf. Section 5).

These facts concern the following four relations with respect to families $\left\{\nu_{\alpha}\right\}_{\alpha \in \mathcal{A}}$ of distributions with zero mean and finite second moment:

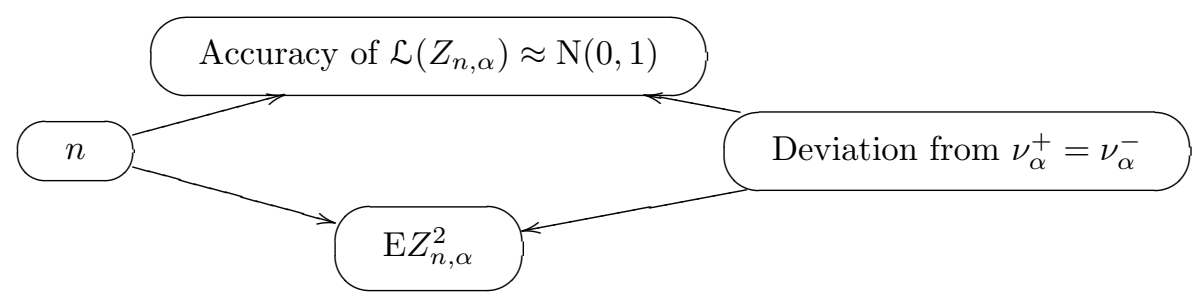

We will in the remaining part of this section consider the asymptotic behaviour as $n \rightarrow \infty$ in the very simple case of $\left\{\nu_{a}\right\}_{a \geq 1}$ with mean-zero two-point distributions:

$$
\nu_{a}=a(1+a)^{-1} \delta_{1}+(1+a)^{-1} \delta_{-a}
$$

Note that $a=1$ is the symmetric case whereas asymmetry increases with $a$.

Suppose we were interested in assessing normal approximation of a specified quantile of $\left(Z_{n}\right)_{a}$ up to a certain degree of accuracy. One could had conjectured there to be an equivalent condition of the form $n \geq n_{0}, \mathrm{E} Z_{n, a}^{2}-1 \leq C$. The two theorems below however show that a condition of the latter kind may omit cases of normal convergence of order $\varepsilon(\log n)^{-1 / 2}$ with $\varepsilon$ depending on the previous degree of accuracy, and that $C$ should be replaced by a sequence $C_{n}$ of asymptotical order $\delta n$ for some $\delta>0$ in order to correspond to the fixed degree of accuracy in the normal approximation.

We also conclude from the theorems below that there are sequences $\left\{a_{n}\right\}$ such that $\left\{Z_{n, a_{n}}\right\}$ converges to $\mathrm{N}(0,1)$ while $\left\{\mathrm{E} Z_{n, a_{n}}^{2}\right\}$ diverges or converges to any number different from 1 . On the other hand, $\mathrm{E} Z_{n, a_{n}}^{2} \rightarrow 1$ implies that $\left\{Z_{n, a_{n}}\right\}$ converges to $\mathrm{N}(0,1)$.

Theorem 6.1. There is a positive constant $C$ such that

$$
\sup _{x}\left|\mathrm{P}\left(Z_{n, a} \leq x\right)-\Phi(x)\right| \leq C \sqrt{a / n}
$$

There are moreover point masses in $Z_{n, a}$ of order $\sim \frac{1}{\sqrt{\pi}} \sqrt{a_{n} / n}$ for sequences $\left\{a_{n}\right\}$ satisfying $a_{n}=\mathrm{o}(n)$ and $a_{n} \rightarrow \infty$.

Proof. The first part is a consequence of the Berry-Esseen theorem for self-normalized sums, Theorem 1.1 in [1] (cf. also the remark succeeding Corollary 1.5.) To apply the theorem, note that $\beta_{3}=\mathrm{E}\left|X_{1, a}\right|^{3}=a\left(1+a^{2}\right) /(1+a)$ and that $\sigma_{3}=\left(\mathrm{E} X_{1, a}^{2}\right)^{3 / 2}=a^{3 / 2}$ so that $\beta_{3} / \sigma_{3}=$ $a^{-1 / 2}\left(1+a^{2}\right) /(1+a) \leq a^{1 / 2}$.

As for the second part, $S_{n, a}$ follows a discrete distribution described by

$$
s_{k}=k-(n-k) a, \quad p_{k}=\left(\begin{array}{l}
n \\
k
\end{array}\right) \frac{a^{k}}{(a+1)^{k}} \frac{1}{(a+1)^{n-k}}, \quad k=0, \ldots, n .
$$

$Z_{n, a}$ is constant over any event $\left\{S_{n, a}=s_{k}\right\}$. Considering point masses $p_{k}$ close to $\left\{S_{n, a}=0\right\}$ leads to an approximate equation $\left(n-k_{n}\right) a=k$. The assumption $a_{n}=\mathrm{o}(n)$ implies that $k_{n} \rightarrow \infty$ and that $\left(n-k_{n}\right) \rightarrow \infty$ so that, by Stirling approximation,

$$
\left(\begin{array}{l}
n \\
k
\end{array}\right) \sim \frac{1}{\sqrt{\pi}} \frac{\sqrt{n}}{\sqrt{n-k} \sqrt{k}} \frac{n^{n}}{(n-k)^{n-k} k^{k}}=\frac{1}{\sqrt{\pi}} \sqrt{a_{n} / n} \frac{a_{n}+1}{a_{n}} \frac{\left(a_{n}+1\right)^{n}}{a_{n}^{k}},
$$


which in turn gives

$$
\left(\begin{array}{l}
n \\
k
\end{array}\right) \frac{a^{k}}{\left(a_{n}+1\right)^{k}} \frac{1}{\left(a_{n}+1\right)^{n-k}} \sim \frac{1}{\sqrt{\pi}} \sqrt{a_{n} / n} \frac{a_{n}+1}{a_{n}} \sim \frac{1}{\sqrt{\pi}} \sqrt{a_{n} / n},
$$

proving the second part of the theorem.

Theorem 6.2. $\mathrm{E} Z_{n, a}^{2}$ is increasing in a for any $n \geq 2$. Moreover, $\lim _{n \rightarrow \infty} \mathrm{E} Z_{n, a_{n}}^{2}=1+\theta$ for $a_{n}=n(\log (n / \theta))^{-1}$ and positive constants $\theta$. Finally, $\mathrm{E} Z_{n, a_{n}}^{2} \sim n e^{-1 / \varepsilon}$ for $a_{n}=\varepsilon n$ and positive constants $\varepsilon$.

Proof. It follows from Theorem 3.1 that

$$
\mathrm{E} Z_{n, a}^{2}-1=n(n-1) \int_{0}^{\infty}\left(\mathrm{E}\left(X_{1, a} e^{-t X_{1, a}^{2}}\right)\right)^{2}\left(\mathrm{E}\left(e^{-t X_{1, a}^{2}}\right)\right)^{n-2} \mathrm{~d} t
$$

Now,

$$
\begin{aligned}
\mathrm{E} X_{1, a} e^{-t X_{1, a}^{2}} & =(a /(a+1))\left(e^{-t}-e^{-t a^{2}}\right), \\
\mathrm{E} e^{-t X_{1, a}^{2}} & =(a /(a+1))\left(e^{-t}+e^{-t a^{2}} a^{-1}\right) .
\end{aligned}
$$

The expressions (16)-(17) are easily checked to be increasing in $a \geq 1$. We conclude that $\mathrm{E} Z_{n, a}^{2}$ is increasing in $a$.

Inserting (16)-(17) into (15) yields

$$
\mathrm{E} Z_{n, a}^{2}-1=n(n-1)(a /(a+1))^{n} \int_{0}^{\infty}\left(e^{-t}-e^{-t a^{2}}\right)^{2}\left(e^{-t}+e^{-t a^{2}} a^{-1}\right)^{n-2} \mathrm{~d} t .
$$

Choosing $a_{n}=n(\log (n / \theta))^{-1}$ gives

$$
\left(\left(a_{n}+1\right) / a_{n}\right)^{n}=(1+\log (n / \theta) / n)^{n} \sim n / \theta,
$$

so that $n(n-1)(a /(a+1))^{n} \sim n \theta$ and

$$
\mathrm{E} Z_{n, a_{n}}^{2}-1 \sim n \theta \int_{0}^{\infty}\left(e^{-t}-e^{-t a_{n}^{2}}\right)^{2}\left(e^{-t}+e^{-t a_{n}^{2}} a_{n}^{-1}\right)^{n-2} \mathrm{~d} t .
$$

Similarly, $a_{n}=\varepsilon n$ yields

$$
\mathrm{E} Z_{n, a_{n}}^{2}-1 \sim n^{2} e^{-1 / \varepsilon} \int_{0}^{\infty}\left(e^{-t}-e^{-t a_{n}^{2}}\right)^{2}\left(e^{-t}+e^{-t a_{n}^{2}} a_{n}^{-1}\right)^{n-2} \mathrm{~d} t .
$$

Evaluating the integral in (18) after expansion of the parentheses gives

$$
\sum_{k=0}^{n-2}\left(\begin{array}{c}
n-2 \\
k
\end{array}\right) a_{n}^{-k} \frac{1}{n+k\left(a_{n}^{2}-1\right)}+\frac{1}{n+(k+2)\left(a_{n}^{2}-1\right)}-\frac{2}{n+(k+1)\left(a_{n}^{2}-1\right)} .
$$

The term $k=0$ is

$$
\frac{1}{n}+\frac{1}{n+2\left(a_{n}^{2}-1\right)}-\frac{2}{n+\left(a_{n}^{2}-1\right)} \sim \frac{1}{n}
$$

for all choices of choices of $\left\{a_{n}\right\}$ at issue, and it hence remains to show that

$$
n \sum_{k=1}^{n-2}\left(\begin{array}{c}
n-2 \\
k
\end{array}\right) a_{n}^{-k} \frac{1}{n+k\left(a_{n}^{2}-1\right)}+\frac{1}{n+(k+2)\left(a_{n}^{2}-1\right)}-\frac{2}{n+(k+1)\left(a_{n}^{2}-1\right)}=\mathrm{o}(1) .(19)
$$

Algebraic manipulations yield

$$
\begin{aligned}
& \frac{1}{n+k\left(a_{n}^{2}-1\right)}+\frac{1}{n+(k+2)\left(a_{n}^{2}-1\right)}-\frac{2}{n+(k+1)\left(a_{n}^{2}-1\right)} \\
= & \frac{2\left(a_{n}^{2}-1\right)^{2}}{\left(n+k\left(a_{n}^{2}-1\right)\right)\left(n+(k+2)\left(a_{n}^{2}-1\right)\right)\left(n+(k+1)\left(a_{n}^{2}-1\right)\right)} \leq \frac{2}{k^{3} a_{n}^{2}},
\end{aligned}
$$


which replaces (19) by

$$
n a_{n}^{-2} \sum_{k=1}^{n-2}\left(\begin{array}{c}
n-2 \\
k
\end{array}\right) a_{n}^{-k} k^{-3}=\mathrm{o}(1) .
$$

Simplify further, by omitting the irrelevant last term on the left hand side and by replacing $a_{n}^{-k}$ and $a_{n}^{-2}$ by the larger $a_{n-2}^{-k}$ and $a_{n-2}^{-2}$ respectively, into

$$
n a_{n}^{-2} \sum_{k=1}^{n-1}\left(\begin{array}{l}
n \\
k
\end{array}\right) a_{n}^{-k} k^{-3}=\mathrm{o}(1) .
$$

We may now, since $1 \leq k \leq n-1$, apply the Stirling approximation

$$
\left(\begin{array}{l}
n \\
k
\end{array}\right) \approx \frac{1}{\sqrt{2 \pi}} \sqrt{\frac{n}{k(n-k)}} \frac{n^{n}}{(n-k)^{n-k} k^{k}},
$$

which replaces (20) by

$$
n a_{n}^{-2} \sum_{k=1}^{n-1} \frac{1}{k^{3+1 / 2}} \frac{n^{k}}{k^{k} a_{n}^{k}} \frac{n^{n-k+1 / 2}}{(n-k)^{n-k+1 / 2}}=\mathrm{o}(1) .
$$

Note that the left hand side decreases by replacing $a_{n}$ with $a_{n}^{\prime}, a_{n} \leq a_{n}^{\prime}$. We therefore merely consider $a_{n}=n(\log (n / \theta))^{-1}$, for some $\theta>0$. Now,

$$
(n /(n-k))^{n-k}=(1+k /(n-k))^{n-k} \leq e^{k},
$$

and it hence suffices to verify that

$$
\frac{(\log n)^{2}}{n} \sum_{k=1}^{n-1} \frac{1}{k^{3+1 / 2}}\left(\frac{e \log (n / \theta)}{k}\right)^{k} \frac{n^{1 / 2}}{(n-k)^{1 / 2}}=\mathrm{o}(1)
$$

which we split into

$$
\begin{gathered}
\frac{(\log n)^{2}}{n} \sum_{k=1}^{\lfloor\log (n / \theta) / e\rfloor} \frac{1}{k^{3+1 / 2}}\left(\frac{e \log (n / \theta)}{k}\right)^{k} \frac{n^{1 / 2}}{(n-k)^{1 / 2}}=\mathrm{o}(1), \\
\frac{(\log n)^{2}}{n} \sum_{k=\lceil\log (n / \theta) / e\rceil}^{\lfloor e \log (n / \theta)\rfloor} \frac{1}{k^{3+1 / 2}}\left(\frac{e \log (n / \theta)}{k}\right)^{k} \frac{n^{1 / 2}}{(n-k)^{1 / 2}}=\mathrm{o}(1), \\
\frac{(\log n)^{2}}{n} \sum_{k=\lceil e \log (n / \theta)\rceil}^{n-1} \frac{1}{k^{3+1 / 2}}\left(\frac{e \log (n / \theta)}{k}\right)^{k} \frac{n^{1 / 2}}{(n-k)^{1 / 2}}=\mathrm{o}(1) .
\end{gathered}
$$

Regarding (21), note that $(\log (n / \theta) e / k)^{k}$ is increasing for $k \leq \log (n / \theta)$, so that the left hand side of (21) is bounded by

$$
\frac{(\log n)^{2}}{e n} \log (n / \theta) e^{2 \log (n / \theta) / e} \frac{n^{1 / 2}}{(n-\log (n / \theta) / e)^{1 / 2}} \sim \frac{(\log n)^{3}}{e \theta^{2 / e}} n^{2 / e-1}=\mathrm{o}(1) .
$$

Regarding (22), note that $(\log (n / \theta) / k)^{k} \leq e^{-k} n / \theta$, so that the left hand side of (22) is bounded by

$$
\frac{(\log n)^{2}}{\theta} \frac{n^{1 / 2}}{(n-e \log (n / \theta))^{1 / 2}} \sum_{k=\lceil\log (n / \theta) / e\rceil}^{\lfloor e \log (n / \theta)\rfloor} \frac{1}{k^{3+1 / 2}} \sim \frac{2}{5 \theta} \frac{e^{5 / 2}-e^{-5 / 2}}{(\log n)^{1 / 2}}=\mathrm{o}(1) .
$$

We finally bound the left hand side of (23) by

$$
\frac{(\log n)^{2}}{n^{1 / 2}} \sum_{k=\lceil e \log (n / \theta)\rceil}^{\infty} \frac{1}{k^{3+1 / 2}}=\mathrm{o}(1),
$$

which completes the proof of Theorem 6.2. 


\section{Statistical estimators of $\mathrm{E} Z_{n}^{2}$}

Assume that $\mathrm{E} Z_{n}^{2}$ is of interest with respect to an unknown mean zero random variable $Y$ and that a sample $\left(x_{1}, \ldots, x_{n}\right)$ from $X=Y+\mu$ is given, for some unknown constant $\mu$. Recall from Theorem 3.1 that the target value is given by

$$
\mathrm{E} Z_{n}^{2}=1+\left(n^{2}-n\right) \int_{0}^{\infty}\left(\mathrm{E}\left(Y e^{-t Y^{2}}\right)\right)^{2}\left(\mathrm{E} e^{-t Y^{2}}\right)^{n-2} \mathrm{~d} t
$$

Estimation of $\mathrm{E} Y e^{-t Y^{2}}$ and $\mathrm{E} e^{-t Y^{2}}$, with $\bar{x}$ denoting the mean of the sample, by

$$
\frac{1}{n} \sum_{i=1}^{n}\left(x_{i}-\bar{x}\right) e^{-t\left(x_{i}-\bar{x}\right)^{2}} \quad \text { and } \quad \frac{1}{n} \sum_{i=1}^{n} e^{-t\left(x_{i}-\bar{x}\right)^{2}}
$$

suggests the following estimator of $\mathrm{E} Z_{n}^{2}$ :

$$
\widehat{\mathrm{E} Z_{n}^{2}}=1+\left(n^{2}-n\right) \int_{0}^{\infty}\left(\frac{1}{n} \sum_{i=1}^{n}\left(x_{i}-\bar{x}\right) e^{-t\left(x_{i}-\bar{x}\right)^{2}}\right)^{2}\left(\frac{1}{n} \sum_{i=1}^{n} e^{-t\left(x_{i}-\bar{x}\right)^{2}}\right)^{n-2} \mathrm{~d} t .
$$

The situation described can be somewhat simplified if the quantity of interest is $\mathrm{E} Z_{n}^{2}$ with respect to $X$. One may then instead consider the estimator

$$
\widehat{\mathrm{E} Z_{n}^{2}}=1+\left(n^{2}-n\right) \int_{0}^{\infty}\left(\frac{1}{n} \sum_{i=1}^{n} x_{i} e^{-t x_{i}^{2}}\right)^{2}\left(\frac{1}{n} \sum_{i=1}^{n} e^{-t x_{i}^{2}}\right)^{n-2} \mathrm{~d} t
$$

\subsection{Some properties of the suggested estimators}

Both (25) and (26) are invariant with respect to sample scaling. Note also that (25) is biased in case $Y \stackrel{d}{=}-Y$ and that $(26)$ is biased in case $X \stackrel{d}{=}-X$, since $\widehat{\mathrm{E} Z_{n}^{2}} \geq 1$ and since $\widehat{\mathrm{E} Z_{n}^{2}}>1$ with positive probability whereas $\mathrm{E} Z_{n}^{2}$ is equal to one by Theorem 3.1 .

The integrals in (25) and (26) may be evaluated as follows:

$$
\begin{aligned}
\int_{0}^{\infty}\left(\sum_{i=1}^{n} y_{i} e^{-t y_{i}^{2}}\right)^{2}\left(\sum_{i=1}^{n} e^{-t y_{i}^{2}}\right)^{n-2} \mathrm{~d} t & =\int_{0}^{\infty} \sum_{i_{1}=1}^{n} \cdots \sum_{i_{n}=1}^{n} y_{i_{1}} y_{i_{2}} e^{-t \sum_{k=1}^{n} y_{i_{k}}^{2}} \mathrm{~d} t \\
& =\sum_{i_{1}=1}^{n} \cdots \sum_{i_{n}=1}^{n}\left(\left(y_{i_{1}} y_{i_{2}}\right) / \sum_{k=1}^{n} y_{i_{k}}^{2}\right),
\end{aligned}
$$

with terms such that $y_{i_{1}}=\cdots=y_{i_{n}}=0$ interpreted as 0 . The number of terms in (27) may be reduced by introducing multinomial coefficients. The resulting number is nevertheless too large for practical purposes. We suggest numerical integration if (25) or (26) are to be evaluated.

The standard "nonparametric bootstrap" approach for estimation of $\mathrm{E} Z_{n}^{2}$ in the two situations above is to compute $\mathrm{E} Z_{n}^{2}$ with respect to the empirical distribution of $x_{1}-\bar{x}, \ldots, x_{n}-\bar{x}$, or $x_{1}, \ldots, x_{n}$ respectively, values typically approximated by the mean of a large number of computed values of $Z_{n}^{2}$ with respect to resampled observations. Theorem 7.1 below shows that the two $\widehat{\mathrm{EZ}} Z_{n}^{2}$ above are closed forms for these bootstrap estimators.

Theorem 7.1. For a real valued vector $\mathbf{y}=\left(y_{1}, \ldots, y_{n}\right)$, set

$$
\theta=1+\left(n^{2}-n\right) \int_{0}^{\infty}\left(\frac{1}{n} \sum_{i=1}^{n} y_{i} e^{-t y_{i}^{2}}\right)^{2}\left(\frac{1}{n} \sum_{i=1}^{n} e^{-t y_{i}^{2}}\right)^{n-2} \mathrm{~d} t .
$$

Let moreover, for $r \geq 1, \mathbf{Y}_{r}=\left(Y_{1, r}, \ldots, Y_{n, r}\right)$ be i.i.d. random vectors with i.i.d. components $Y_{k, r}$ uniformly distributed on $\left\{y_{1}, \ldots, y_{n}\right\}$ and set, for $N \geq 1, \theta_{N}=\frac{1}{N} \sum_{r=1}^{N} Z_{n, r}^{2}$ with

$$
Z_{n, r}^{2}= \begin{cases}\left(\sum_{i=1}^{n} Y_{i, r}\right)^{2} / \sum_{i=1}^{n} Y_{i, r}^{2}, & \mathbf{Y}_{r} \neq \mathbf{0} \\ 0, & \mathbf{Y}_{r}=\mathbf{0}\end{cases}
$$

It follows that $\lim _{N \rightarrow \infty} \theta_{N} \stackrel{\text { a.s. }}{=} \theta$. 
Proof. Note that

$$
\theta_{N}=1+2 \sum_{1 \leq i<j \leq n} \frac{1}{N} \sum_{r=1}^{N}\left(Y_{i, r} Y_{j, r} / \sum_{k=1}^{n} Y_{k, r}^{2}\right)
$$

and that, for any $1 \leq i<j \leq n$, due to the law of large numbers,

$$
\lim _{N \rightarrow \infty} \frac{1}{N} \sum_{r=1}^{N}\left(Y_{i, r} Y_{j, r} / \sum_{k=1}^{n} Y_{k, r}^{2}\right) \stackrel{a . s .}{=} \frac{1}{n^{n}} \sum_{i_{1}=1}^{n} \cdots \sum_{i_{n}=1}^{n}\left(y_{i_{1}} y_{i_{2}} / \sum_{k=1}^{n} y_{i_{k}}^{2}\right),
$$

since $\left\{Y_{i, r} Y_{j, r} / \sum_{k=1}^{n} Y_{k, r}^{2}\right\}_{r \geq 1}$ are independent and identically distributed, assuming the $n^{n}$ (nondistinct) values $y_{i_{1}} y_{i_{2}} / \sum_{k=1}^{n} y_{i_{k}}^{2}$ each with probability $n^{-n}$ (interpreted as 0 for the cases $y_{i_{1}}=$ $\cdots=y_{i_{n}}=0$ by the definition of $Z_{n, r}^{2}$ ). We conclude from (28) and (29) that

$$
\lim _{N \rightarrow \infty} \theta_{N} \stackrel{\text { a.s. }}{=} 1+\left(n^{2}-n\right) \frac{1}{n^{n}} \sum_{i_{1}=1}^{n} \cdots \sum_{i_{n}=1}^{n}\left(y_{i_{1}} y_{i_{2}} / \sum_{k=1}^{n} y_{i_{k}}^{2}\right) .
$$

The desired conclusion follows from (27), (30) and the definition of $\theta$.

\section{Appendix: Characterizing $\lim _{m \rightarrow \infty} Z_{n, m}^{2} \stackrel{d}{=} 1$}

As before, let $\left\{F_{m}\right\}_{m \geq 1}$ be a sequence of cumulative distribution functions all continuous at the origin, let $X_{1, m}, \ldots, \bar{X}_{n, m}$ be independent random variables each with distribution function $F_{m}$ and define

$$
Z_{n, m}^{2}=\left(\sum_{i=1}^{n} X_{i, m}\right)^{2} / \sum_{i=1}^{n} X_{i, m}^{2}, \quad T_{n, m}^{2}=(n-1) Z_{n, m}^{2} /\left(n-Z_{n, m}^{2}\right) .
$$

Theorem 8.1 and Examples 8.1-8.2 reveal that there for any $n \geq 2$ are distribution functions with corresponding $Z_{n}^{2}$ arbitrarily close to 1 in distribution. The same is consequently true with $Z_{n}^{2}$ replaced by $T_{n}^{2}$ (confer the second equation above). Regarding our previous occupations with $\mathrm{E} Z_{n}^{2}$, condition (i) of Theorem 8.1 implies that $\lim _{m \rightarrow \infty} \mathrm{E} Z_{n, m}^{2}=1$. To verify this, note that $Z_{n, m}^{2} \leq n$ by the Cauchy-Schwarz inequality, then confer Theorems 4.4 and 5.9 in [7, Chapter 5].

An interesting description of the limiting behaviour at issue in Theorem 8.1 is given by condition (ii): out of two independent observations from the distribution, the one with largest absolute value completely dominates the other. We remark that two-point distributions symmetric around the origin fulfill the very opposite of condition (ii), $\min \left(\left|X_{1}\right|,\left|X_{2}\right|\right) / \max \left(\left|X_{1}\right|,\left|X_{2}\right|\right)=1$. It is therefore perhaps reasonable to think of the captured behaviour in terms of "explosion of skewness" or "explosion of kurtosis" (confer also the two characteristic examples below).

Theorem 8.1. For sequences $\left\{F_{m}\right\}$ and any fixed integer $n \geq 2$ the following are equivalent:

(i) $\lim _{m \rightarrow \infty} Z_{n, m}^{2} \stackrel{d}{=} 1$;

(ii) $\lim _{m \rightarrow \infty} \min \left(\left|X_{1, m}\right|,\left|X_{2, m}\right|\right) / \max \left(\left|X_{1, m}\right|,\left|X_{2, m}\right|\right) \stackrel{d}{=} 0$;

(iii) $\lim _{m \rightarrow \infty} \sup _{x>0}\left|F_{m}\left(\lambda_{2} x\right)-F_{m}\left(\lambda_{1} x\right)\right|=0 \quad$ for $\quad 0<\lambda_{1} \leq \lambda_{2}<\infty$ and $-\infty<\lambda_{1} \leq \lambda_{2}<0$.

Before turning to the proof of Theorem 8.1, let us mention the following, perhaps most wellknown type of families $\left\{F_{m}\right\}$ satisfying the conditions of Theorem 8.1.

Example 8.1. Distribution functions corresponding to Gamma-distributions with fixed scale parameter and shape parameter converging to zero, i.e. for instance $\{\Gamma(1 / m, \theta)\}$ with $\theta$ fixed.

Example 8.2. Distribution functions corresponding to symmetrized Gamma-distributions with fixed scale parameter and shape parameter converging to zero. (We say that $Z$ follows a symmetrized $\nu$-distribution if $Z \stackrel{d}{=} X-Y$ with $X$ and $Y$ independent and $\nu$-distributed). 
A proof of these two statements will not be given. It is however not hard to see that condition (iii) is satisfied by considering the gamma density functions. In brief, the essential fact is that the density functions approach the behaviour of $f(x)=1 / x$.

Proof of Theorem 8.1. To see that (ii) implies (i), note that

$$
\begin{aligned}
\left|X_{1} X_{2}\right| / \sum_{i=1}^{n} X_{i}^{2} & \leq\left|X_{1} X_{2}\right| / \max \left(X_{1}^{2}, X_{2}^{2}\right) \\
& =\min \left(\left|X_{1}\right|,\left|X_{2}\right|\right) / \max \left(\left|X_{1}\right|,\left|X_{2}\right|\right) .
\end{aligned}
$$

Combined with Cramér's theorem, assumption (ii) therefore gives the desired

$$
Z_{n, m}^{2}-1=2 \sum_{1 \leq i<j \leq n} X_{i, m} X_{j, m} / \sum_{l=1}^{n} X_{l, m}^{2} \stackrel{d}{\longrightarrow} 0 \quad \text { as } \quad m \rightarrow \infty
$$

Regarding the implication (iii) $\Rightarrow$ (ii), set $Y_{1}=\left|X_{1, m}\right|, Y_{2}=\left|X_{2, m}\right|$ and denote their common distribution function by $G$. For any $\lambda>1$,

$$
\begin{aligned}
& \mathrm{P}\left(Y_{1} \leq \lambda Y_{2}\right)-1 / 2 \leq \mathrm{P}\left(Y_{1} \leq \lambda Y_{2}\right)-\mathrm{P}\left(Y_{1}<Y_{2}\right)=\int_{0}^{\infty} \mathrm{P}\left(Y_{1} \leq \lambda x\right)-\mathrm{P}\left(Y_{1}<x\right) \mathrm{d} G(x) \\
\leq & \sup _{x>0}\left\{F_{m}(\lambda x)-F_{m}(x)\right\}+\sup _{x>0}\left\{F_{m}(-x)-F_{m}(-\lambda x)\right\} .
\end{aligned}
$$

Condition (iii) hence implies that $\lim _{m \rightarrow \infty} \mathrm{P}\left(Y_{1} \leq \lambda Y_{2}\right)=1 / 2$, for each $\lambda>1$. We conclude that (ii) holds, since for any $0<\varepsilon<1$,

$$
\mathrm{P}\left(\min \left(Y_{1}, Y_{2}\right) / \max \left(Y_{1}, Y_{2}\right) \geq \varepsilon\right)=2 \mathrm{P}\left(Y_{1} \leq \varepsilon^{-1} Y_{2}\right)-1 .
$$

It remains to verify that (i) $\Rightarrow$ (iii). Assume that (iii) does not hold, i.e. that there exists a subsequence $\left\{m_{k}\right\}$, scalings $\left\{x_{k}\right\}$ and constants $\varepsilon>0, \lambda_{1} \leq \lambda_{2}$ such that $\left|F_{m_{k}}\left(\lambda_{2} x\right)-F_{m_{k}}\left(\lambda_{1} x\right)\right|>$ $\varepsilon$. We conclude that

$$
\mathrm{P}\left(2 \sum_{1 \leq i<j \leq n} X_{i, m_{k}} X_{j, m_{k}} / \sum_{l=1}^{n} X_{l, m_{k}}^{2}>(n-1) \min \left(\lambda_{1}^{2}, \lambda_{2}^{2}\right) / \max \left(\lambda_{1}^{2}, \lambda_{2}^{2}\right)\right) \geq \varepsilon^{n}
$$

and that (i) cannot hold.

\section{Acknowledgement}

Professor Svante Janson generously communicated the main idea behind the fundamental Lemma 3.1. My PhD supervisors Allan Gut and Silvelyn Zwanzig rendered useful critizism of many kinds.

\section{References}

[1] Bentkus, V. And Götze, F. (1996). The Berry-Esseen bound for Student's statistic. Ann. Probab. 24, 491-503.

[2] Billingsley, P. (1999). Convergence of Probability Measures, 2nd edition. John Wiley and Sons.

[3] Cohn, D. L. (1980). Measure Theory. Birkhäuser.

[4] Cressie, N. (1980). Relaxing assumptions in the one sample t-test. Austral. J. Statist. 22, $143-153$

[5] Efron, B. (1969). Student's t-test under symmetry conditions. J. Amer. Statist. Assoc. 64, 1278-1302. 
[6] Giné, E., Götze, F. And Mason, D.M. (1997). When is the student $t$-statistic asymptotically standard normal? Ann. Probab. 25, 1514-1531.

[7] Gut, A. (2007). Probability: A Graduate Course, Corr. 2nd printing. Springer-Verlag.

[8] Posten, H.O. (1979). The robustness of the one-sample $t$-test over the Pearson system. $J$. Statist. Comput. Simul. 9, 133-149.

[9] Rudin, W. (1976). Principles of Mathematical Analysis, 3rd edition. McGraw-Hill Book Co.

[10] ZABELl, S.L. (2008). On Student's 1908 article "The probable error of a mean". With comments and a rejoinder by the author. J. Amer. Statist. Assoc. 481, 1-20.

Fredrik Jonsson, Department of Mathematics, Uppsala University, Box 480, SE-751 06 Uppsala, Sweden. Email: jonsson@math.uu.se

URL: http://www.math.uu.se/ jonsson 